\title{
O.S.P.
}

L'orientation scolaire et professionnelle

$29 / 2 \mid 2000$

Diplôme et marché du travail

\section{Conception du diplôme et formes d'usage du CAP}

Design of the diploma and use of the CAP

Éric Cahuzac, Fabienne Maillard et Maurice Ourtau

\section{(2) OpenEdition}

Journals

Édition électronique

URL : http://journals.openedition.org/osp/5954

DOI : 10.4000/osp.5954

ISSN : 2104-3795

Éditeur

Institut national d'étude du travail et d'orientation professionnelle (INETOP)

Édition imprimée

Date de publication : 15 juin 2000

ISSN : 0249-6739

Référence électronique

Éric Cahuzac, Fabienne Maillard et Maurice Ourtau, « Conception du diplôme et formes d'usage du CAP », L'orientation scolaire et professionnelle [En ligne], 29/2 | 2000, mis en ligne le 04 juin 2018,

consulté le 16 décembre 2020. URL : http://journals.openedition.org/osp/5954 ; DOI : https://doi.org/ 10.4000/osp.5954

Ce document a été généré automatiquement le 16 décembre 2020.

(c) Tous droits réservés 


\section{Conception du diplôme et formes d'usage du CAP}

Design of the diploma and use of the CAP

Éric Cahuzac, Fabienne Maillard et Maurice Ourtau

\section{Introduction}

1 Institué en 1919 le certificat d'aptitude professionnelle (C.A.P.) a d'abord été un diplôme départemental, voire local ${ }^{1}$. C'est seulement en 1943 qu'il devient un diplôme national. Le premier règlement général des C.A.P. industriels date de 1950 . L'intégration de l'enseignement professionnel au sein du système scolaire assigne au C.A.P. une fonction de scolarisation dans le dispositif de formation de masse.

2 La création des collèges d'enseignement technique (C.E.T.) en 1959 et la décision (qui deviendra effective en 1967) de prolonger jusqu'à 16 ans la scolarité obligatoire, sont deux événements qui ont renforcé cette fonction nouvelle.

3 À partir de 1982 l'orientation à l'issue de la classe de cinquième n'est plus obligatoire, elle est soumise à une acceptation de la part des élèves et de leur famille ${ }^{2}$. De plus en plus le C.A.P. apparaît comme un diplôme réservé aux jeunes en difficulté. Son éviction du système scolaire est programmée avec la création du baccalauréat professionnel en 1985.

4 Parallèlement à cette politique deux décisions ont conforté la position du C.A.P. comme certification connue et reconnue sur le marché du travail : la révision des B.E.P. et leur recentrage sur de grands domaines professionnels, la politique d'association des B.E.P. aux C.A.P. traditionnels qui encourage les élèves de B.E.P. à se présenter également aux épreuves des C.A.P. associés.

5 À la fin des années 80 la mise en oeuvre de ces politiques successives suscitait toujours des débats sur le maintien ou la disparition du C.A.P. La place du C.A.P. dans le système des diplômes était ainsi l'une des " questions vives " posées dans le rapport au Secrétariat d'État à l'Enseignement Technique (Tanguy, 1991, p.109). La redéfinition du 
C.A.P. était considérée à l'époque comme une nécessité « consécutive à la technicisation du B.E.P. et sa propension à devenir propédeutique ».

6 Des phénomènes marquants ont ensuite eu lieu dans la dernière décennie : l'aggravation du chômage des jeunes, l'affirmation du rôle «propédeutique » du B.E.P. (Fourcade \& Ourtau, 1999), le rôle prépondérant des petites entreprises dans les recrutements, l'usage effectif du baccalauréat professionnel sur le marché du travail.

7 La question de la relance du C.A.P. dans le système scolaire ${ }^{3}$ intervient dans ce contexte turbulent, alors que ce diplôme a subi un fort discrédit de la part de l'institution scolaire mais aussi de la part des entreprises, qui n'ont jamais cessé de le réclamer tout en recrutant abondamment des jeunes plus diplômés.

8 Cette contribution tente de faire le point sur les évolutions du C.A.P. et d'observer ses différentes formes d'usage dans les espaces de formation et d'emploi. L'approche historique du processus de création, suppression, rénovation des C.A.P. témoigne de la capacité d'adaptation et d'évolution d'un diplôme professionnel dans sa fonction première de préparation pour l'accès à un emploi. Cette évolution, qui suit très largement celle de l'organisation du travail (C.A.P. de métiers, C.A.P. transversaux, approche compétence, ...) est le résultat de confrontation des points de vues et des intérêts de divers acteurs (pouvoirs publics, salariés, patronat) au sein des instances de concertation spécialisées (point 1).

9 L'hétérogénéité de l'offre actuelle de formation et de certification en C.A.P. (contenu de la formation, niveau d'exigence requis pour obtenir le diplôme, publics en formation, candidatures à l'examen), abordée dans les points 2 et 3, est pour partie le résultat de confrontations successives qui opposent souvent des logiques contradictoires nécessitant la construction de compromis difficiles à gérer.

10 Les deux derniers points ${ }^{4}$ traitent de la situation du C.A.P. sur le marché du travail dans une perspective inter-générationnelle et un contexte de hausse continue des niveaux d'éducation qui tend à réduire le champ des emplois accessibles aux titulaires du seul C.A.P. confrontés à la concurrence de diplômés de niveaux plus élevés et à aggraver leurs conditions d'entrée en activité.

\section{Un système de formation et de certification en évolution continue}

11 L'observation de l'histoire du C.A.P. à travers ses spécialités depuis 1944, c'est-à-dire au moment où la scolarisation de l'enseignement technique se met en place, montre que le mouvement de création/rénovation des C.A.P. ne s'est jamais interrompu au cours des années, même lorsque de lourdes menaces pesaient sur l'avenir de ce diplôme. La création du B.E.P. ${ }^{5}$, qui aurait pourtant dû remplacer à terme le C.A.P., s'est ainsi accompagnée de multiples créations-rénovations de C.A.P., aussi bien dans le domaine industriel que dans les services. C.A.P. et B.E.P. du tertiaire administratif ont ainsi pu longtemps coexister tout en visant les mêmes emplois. La division C.A.P./B.E.P. ne renvoie en conséquence à aucune distinction sectorielle ou professionnelle immédiate, les deux diplômes ayant suivi des chemins identiques malgré leurs ambitions officiellement différentes (le B.E.P. devait concerner un champ d'activité plus large que celui du C.A.P., c'est pourquoi il était prévu de créer un nombre limité de B.E.P.). En fait les B.E.P. se sont très vite multipliés, sans pour autant que les C.A.P. disparaissent. C'est 
leur public scolaire plus que leur définition et leur contenu qui a finalement distingué les deux diplômes, les B.E.P. accueillant des jeunes issus de la troisième de collège, les C.A.P. formant les élèves orientés après la cinquième. Cette distinction par les publics scolaires, qui est en fait une distinction par les plus ou moins grandes difficultés scolaires des jeunes, a pris un tel caractère d'évidence et de nécessité au fil du temps qu'on a créé en parallèle ou successivement des C.A.P. et des B.E.P. sanctionnant le même contenu de formation et finalisés sur les mêmes emplois. Dans certains cas, le même intitulé les désigne (C.A.P. Vente et B.E.P. Vente par exemple ; la distinction repose ici sur les publics et les modes de formation : le C.A.P. concerne les apprentis, le B.E.P. s'adresse surtout aux élèves).

On peut identifier trois grandes périodes dans l'histoire récente du C.A.P. :

\section{La multiplication des C.A.P. de métier et des options de spécialisation (1944 - fin des années 60)}

Les C.A.P. créés jusqu'à la fin des années 60 sont très orientés sur des métiers, conçus en fonction d'une division du travail particulièrement rigide, aussi bien dans l'industrie que dans le tertiaire administratif (plusieurs spécialités sont proposées, par exemple, pour les emplois du secrétariat). Les C.A.P. industriels sont nombreux, principalement dans la métallurgie. Ils accompagnent la mise en place de l'industrie moderne, dont les besoins en ouvriers qualifiés sont importants, et se décomposent en spécialités très fines qui correspondent à une organisation taylorisée du travail. Malgré la tertiarisation de l'économie, peu de C.A.P. s'adressent au secteur des services, à l'exception des C.A.P. d'Hôtellerie-restauration, du C.A.P. Coiffure et d'un C.A.P. Aide maternelle, créé en 1949 et supprimé en 1975.

Dans le même moment, le concours d'entrée qui permet l'accès à la formation est supprimé, et le C.A.P. se voit assigner une fonction scolaire d'accueil pour les publics évincés de l'enseignement secondaire général. Toutefois, dans la mesure où le plein emploi favorise l'insertion des diplômés et leur mobilité, le C.A.P. continue à jouer un rôle de promotion sociale et professionnelle pour ses titulaires.

La durée de vie des C.A.P. créés pendant cette phase est de vingt à trente ans. L'actualisation des formations n'est donc pas un mot d'ordre, sauf pour quelques spécialités qui subissent plusieurs rénovations en quelques années (Chaudronnier, Électromécanicien, Monteur en installations téléphoniques, tous les C.A.P. de la sidérurgie comme ceux du textile et de l'habillement ; les industries en difficulté reconstruisent régulièrement leurs diplômes).

Au cours des années 60 , on met en place beaucoup de spécialisations, d'options, à partir de C.A.P. existants. Plus que les C.A.P. autonomes, ce sont les options diverses qui se multiplient (on crée par exemple 3 options au C.A.P. Chaudronnier...). Les intitulés des diplômes indiquent le cœur du métier, tandis que les options renvoient à ses différents segments et à une division très figée du travail. Si toutes ces spécialisations satisfont la demande des branches professionnelles, elles se réfèrent à une définition du métier que l'administration de l'Éducation nationale invaliderait aujourd'hui. Le morcellement de chaque métier en plusieurs spécialités apparait ainsi très réducteur et peu compatible avec l'évolution des organisations du travail dans les entreprises. 
17 Le dénombrement des C.A.P. est très difficile à faire pour cette période : alors que B. Charlot et M. Figeat (1985) parlent de «balkanisation » des C.A.P., G. Brucy recense en 1961234 C.A.P. nationaux, auxquels s'ajoutent 264 C.A.P. régionaux ou départementaux ${ }^{6}$. Dans la mesure où il existait 2314 C.A.P. en 1942, cet ensemble d'environ 500 C.A.P. montre qu'un gros effort de rationalisation de l'offre de diplômes a été mené ${ }^{7}$.

\section{Des innovations radicales (1970 - milieu des années 80)}

Malgré les menaces qui pèsent alors sur le C.A.P. et son identification aux métiers les plus traditionnels, c'est peu après la création du B.E.P. que sont constitués les C.A.P. les plus innovants par rapport à l'histoire de ce diplôme, au sens où ils rompent avec ce qui avait fait jusqu'alors la tradition en matière de définition de spécialités. Ces diplômes intègrent ainsi une dimension transversale qui devrait pourtant être exclusive au B.E.P. Des C.A.P. de conduite de système industriel sont créés en 1969 dans l'industrie du bois, et un C.A.P. " fonctions de l'informatique » est instauré en 1970. Plusieurs diplômes sont institués dans le domaine des services et de nouvelles spécialités industrielles voient le jour comme l'Électrotechnique (CAP. à 5 options). Cependant, les C.A.P. restent en général très spécialisés, qu'ils concernent les emplois du tertiaire administratif, les emplois des services aux collectivités ou « à la personne », les emplois artisanaux ou industriels. Malgré l'émergence de nouvelles spécialités et la croissance de nouveaux secteurs d'activité, les mêmes types de normes semblent régir la construction des C.A.P. L'offre de C.A.P. n'est pas une offre d'anticipation, comme elle tente de l'être depuis le milieu des années quatre-vingts, mais plutôt une offre de réponse aux besoins énoncés par les entreprises et par les branches professionnelles.

Les intitulés des diplômes changent, mettant en valeur l'emploi et non plus l'activité. Le « conducteur » remplace ainsi la " conduite », qui le remplacera à nouveau quelques années plus tard. Les C.A.P. sont des diplômes "d'agent... ", de " cartonnier ", de " mécanicien ", de " préparateur ", etc. Les nouveaux titres sont souvent liés à la propagation des nouvelles technologies, mais leur déclinaison extrêmement précise donne une image rétrospectivement très cloisonnée des diplômes qu'ils désignent. C'est après 1984 qu'un mouvement inverse se dessine, puisqu'à ces titres se substituent des intitulés plus génériques, qui évoquent l'activité principale visée par le diplôme : « vente ", « ennoblissement textile ", « laminage », « peinture ", « mise en œuvre des plastiques »...

Alors que le C.A.P. est voué à disparaitre dans le système scolaire, il est néanmoins préparé par 475528 élèves en 1970/71, face à 134440 élèves en formation au B.E.P...

\section{Un mouvement permanent de suppression et de rénovation (de 1984 à nos jours)}

21 Au début des années 80 le C.A.P. est à nouveau explicitement condamné, car il n'attire pas les élèves, répond modérément aux attentes des entreprises, paraît inadapté à l'évolution de la structure des emplois et à leur contenu, et parce qu'il occupe une place équivoque dans le projet d'incitation à la poursuite d'études du gouvernement de l'époque. Pour lui conserver une place dans l'offre de diplômes et lui faire jouer un rôle utile, on le définit comme une certification et non plus comme une formation, et l'on crée - ou l'on rénove - des C.A.P. expressément en fonction des publics qui en auront 
l'usage : jeunes des sections d'éducation spécialisée, salariés non qualifiés, apprentis... Correspondant au premier niveau de qualification professionnelle dans le système d'emploi, le C.A.P. devient le plus petit diplôme existant, indispensable mais très insuffisant : parce qu'il est indispensable, son existence est maintenue, mais son insuffisance et sa faible attractivité incitent à le repousser hors des frontières de l'école. Le C.A.P. se voit alors attribuer plusieurs fonctions sociales : permettre aux jeunes en grandes difficultés d'accéder à un diplôme, sanctionner les formations de métier dispensées en apprentissage, favoriser l'accès à la qualification ou la reconversion d'adultes en difficultés. C.A.P. et publics en difficultés sont nettement associés, sans plus aucune considération d'âge ni de statut.

C'est en 1984 que naissent les C.A.P. transversaux de l'industrie institués dans une perspective de qualification d'ouvriers spécialisés. Ce sont des diplômes liés au développement des nouveaux systèmes de production, conçus dans une optique de grande polyvalence et constitués à partir d'une logique de compétences qui rompt avec les logiques de métier et de savoir-faire qui l'emportaient auparavant dans les formations industrielles. Les nouveaux procès de production et les nouvelles organisations du travail sont clairement pris en compte dans ces nouveaux diplômes, mais leur caractère transversal est encore relatif, dans la mesure où les diplômes sont toujours définis en fonction du segment d'activité et/ou des produits auxquels destinent les C.A.P. (ce qui n'est plus le cas avec le C.A.P. « Exploitation d'Installations Industrielles ", créé en 1994).

Toute cette période est traversée par de nombreux mouvements : créations, rénovations, créations suivies de près d'abrogations, suppressions. La politique ambivalente à l'égard du C.A.P. s'observe nettement dans ces évolutions parfois très rapides qui interviennent, et dont certaines sont contradictoires (dans la mécanique par exemple, les créations-rénovations-suppressions se succèdent à un rythme soutenu, et la durée de vie des diplômes raccourcit de manière considérable). Des diplômes sont abrogés après une seule session, sans être nécessairement supprimés pour autant et sans que l'on puisse d'emblée identifier les raisons de leur abrogation immédiate. Certains diplômes, créés en 1988 pour prendre la place de diplômes jugés obsolètes, sont supprimés peu après les premières sessions d'examen : ainsi, dans la mécanique industrielle, le tertiaire administratif, l'industrie textile mais aussi les industries graphiques, des C.A.P. récents ou rénovés disparaissent après quelques années d'existence, ou bien doivent être prochainement supprimés (I'15.I.M.M. demande en effet depuis quelques années que soient supprimés tous les C.A.P. liés à la mécanique et au travail des métaux). C'est dans ces secteurs que les suppressions les plus définitives ont eu lieu, puisqu'il n'existe plus aucun C.A.P. dans la sidérurgie, le tertiaire de bureau, la mécanique et l'industrie textile, en raison de la disparition progressive des emplois d'exécution auxquels préparait le C.A.P. Dans les autres spécialités, les abrogations désignent surtout des reconstitutions. En effet, si un grand nombre de C.A.P. ont disparu depuis 1944, faisant passer le nombre des spécialités de près de 350 dans les années 70 à 220 en 1999, de nombreuses suppressions réalisées ont en fait souvent consisté en la reconstruction d'un diplôme à partir de plusieurs C.A.P. existants (5 C.A.P. deviennent un CAP. à 5 options).

Les mouvements les plus radicaux interviennent dans cette dernière période, aussi bien en termes de suppression que de création de diplômes. Les C.A.P. conçus pour répondre aux besoins en qualification de personnel salarié se démultiplient, et la définition des 
diplômes à partir d'une logique de « compétences » substituées aux savoir-faire prend forme : l'adaptabilité, la polyvalence, le sens des initiatives.., prennent le pas sur les savoir-faire, qui apparaissent comme des composantes secondaires de ces compétences ; on définit un comportement professionnel que l'on décline ensuite en connaissances et en pratiques à acquérir. Ce n'est pas le métier qui détermine la forme de ces nouveaux C.A.P. mais un type particulier d'organisation du travail, fondé sur la flexibilité, l'interchangeabilité des personnels et leur autonomie.

Plusieurs C.A.P. sont créés en liaison avec des emplois émergents ou en voie de professionnalisation : gardien d'immeuble, gestion des déchets, livreur, agent de prévention et de médiation... En outre, l'espérance de vie des diplômes n'est plus la même qu'autrefois puisqu'il est prévu de les rénover tous les cinq ans. 220 C.A.P. restent présents dans l'offre de diplômes, et malgré les interrogations que suscite l'avenir de cette certification, le mouvement de création-recomposition de cette offre ne s'est pas interrompu.

Mise en oeuvre dans le cadre plus global de la restructuration du système d'enseignement professionnel, la refonte du dispositif de formation et de certification au C.A.P. a eu des effets importants. Le C.A.P. a fini peu à peu par quitter le système scolaire pour se voir réservé à l'apprentissage, au point que les élèves n'étaient plus que 19266 en 1997/98 à être inscrits en formation à un C.A.P. en 3 ans, et 46263 à préparer un C.A.P. en 2 ans. Parallèlement, le B.E.P. a vu gonfler ses effectifs, qui sont passés de 134440 lycéens en 1970/ 71 à 467704 en 1997/98 (France métropolitaine, public + privé) ${ }^{8}$. Comme l'envisageaient ses concepteurs, le B.E.P. a pris la place du C.A.P. dans le système scolaire, mais il a fallu près de trente ans pour que cette substitution aboutisse. Par ailleurs, c'est plus en tant que formation préparatoire au baccalauréat professionnel qu'en tant que diplôme d'insertion professionnelle que le B.E.P. occupe cette place majeure.

\section{L'état actuel de l'offre de formation et de la certification en C.A.P.}

27 Le C.A.P. désigne désormais un ensemble de diplômes très hétérogène, tant par le niveau d'exigences requis pour l'obtention du diplôme, par le contenu de la formation que par le type de public en formation ou candidat à l'examen. Il joue un rôle pluriel auprès de populations très diverses, composées de jeunes en difficultés évincés des préparations au B.E.P. mais qui restent dans le système scolaire, d'apprentis de niveaux très différents (des bacheliers à l'entrée en C.A.P. Employé en pharmacie, des jeunes peu conformes au modèle scolaire en C.A.P. Peinture), d'adultes en formation continue ou d'élèves en formation au B.E.P. qui obtiennent facilement le C.A.P. grâce à l'association des deux diplômes. Cette multiplicité des rôles accordés au C.A.P. fait que le nombre des candidats aux différents C.A.P. est toujours plus élevé que celui des inscrits en dernière année de formation. Il arrive même que des C.A.P. n'existent pas en tant que formation mais seulement en tant que certification, à l'usage des élèves de B.E.P. et des candidats issus de la formation continue...

Dans l'ensemble des C.A.P. officiellement en activité, une quarantaine seulement concerne des populations de formés supérieures à 500 inscrits. Certains n'ont même aucun inscrit en formation ni aucun candidat au diplôme en 1998. Il peut s'agir de C.A.P. "réservés " à la formation continue ou de C.A.P. qui jouent un rôle de 
conservation des métiers d'art. Des C.A.P. connaissent également de tels problèmes de recrutement qu'ils ont une existence hasardeuse. C'est le cas par exemple de plusieurs C.A.P. industriels très spécialisés (métallurgie et chimie) et de quelques C.A.P. du bâtiment et des travaux publics.

\section{Les lycées et les centres de formation d'apprentis préparent à des spécialités différentes}

L'offre de formation est divisée entre la voie scolaire et l'apprentissage, puisque les spécialités préparées dans l'un et l'autre système de formation ne sont presque jamais les mêmes. Les lycées et les centres d'apprentis se polarisent sur des spécialités distinctes, comme le montre ci-dessous la liste des dix plus importants C.A.P. dans chaque système de formation :

TABLEAU 1. Les dix premiers C.A.P. d'apprentis / les dix premiers C.A.P. scolaires

\begin{tabular}{|c|c|c|c|c|}
\hline $\begin{array}{l}\text { (EFFECTIFS 1997/98, } \\
\text { Ire ET } 2^{\text {e }} \text { ANNÉE DE FORMATION) }\end{array}$ & & & $\begin{array}{l}\text { (FLUX EN Ire ANNÉE DE FORMATION, } \\
\text { RENTRÉE } 1998 \\
\text { ENSEIGNEMENT PUBLIC ET PRIVÉ) }\end{array}$ & \\
\hline Coiffure & 15 & 075 & Coiffure 3 & 046 \\
\hline Cuisine & 14 & 957 & Esthétique 2 & 081 \\
\hline Vente & 13 & 321 & Employé tech. de collectivité 1 & 910 \\
\hline Maintenance de véhicules & 11 & 124 & Ebéniste 1 & 035 \\
\hline Pâtissier & 10 & 713 & Prêt à porter & 942 \\
\hline Restaurant & 9 & 236 & Vente & 827 \\
\hline Construction-maçonnerie & 8 & 953 & Métallerie & 802 \\
\hline Boulangerie & 8 & 417 & Installations en équipements élect. & 799 \\
\hline Menuiserie & 6 & 941 & Menuiserie & 707 \\
\hline Carrosserie-réparation & 5 & 351 & Agent technique d'aliment. & 696 \\
\hline
\end{tabular}

TABLE 1. The first ten apprenticeship C.A.P. / the first leu scholastic C.A.P.

Trois C.A.P. occupent une place influente dans le système scolaire comme dans le système d'apprentissage : Coiffure, Vente, Menuiserie. Ces spécialités ont des flux importants dans chacun des deux systèmes de formation et les diplômés se livrent une concurrence intense sur le marché du travail. À cette concurrence interne s'ajoute, pour la Vente et la Menuiserie, la concurrence avec les titulaires du B.E.P., désormais perçus par les employeurs comme détenteurs d'un meilleur niveau de formation que les titulaires de C.A.P. La proximité entre C.A.P. et B.E.P. dans une même spécialité, qui 
a conduit à réserver le diplôme de fin d'études, autrement dit le C.A.P., à la population des jeunes en grandes difficultés scolaires, génère une rivalité entre les deux diplômes toujours défavorable au C.A.P. C'est par son public et non par les compétences qu'il sanctionne ou par l'activité qu'il vise qu'est d'abord identifié le C.A.P., caractéristique négative qui tend à occulter sa valeur professionnelle. Néanmoins, comme les jeunes formés en B.E.P. obtiennent mieux que les autres le C.A.P., lorsque celui est associé à leur B.E.P., la supériorité du B.E.P. sur le C.A.P. ne semble pas relever d'une simple représentation et paraît en conséquence difficile à infirmer.

Alors que certains C.A.P. relèvent exclusivement de l'apprentissage, d'autres n'existent que dans les lycées. Sur un total de 220 C.A.P. officiellement répertoriés, 248 sont préparés en apprentissage (des C.A.P. abrogés continuent de vivre pendant quelques années), tandis que 139 sont proposés aux élèves dans le système scolaire.

On remarque également que peu de formations sont réellement mixtes puisque 64 C.A.P. sont des diplômes essentiellement masculins, alors que moins d'une trentaine accueillent surtout des filles. L'apprentissage est bien plus masculin que féminin, en raison des métiers auxquels il prépare et qui sont encore considérés comme des " métiers d'hommes ", peu ouverts à la présence des filles, comme ceux du bâtiment et de l'alimentation (boucher, charcutier, boulanger...). L'offre de diplômes est de toute façon moins ouverte aux filles qu'aux garçons, qui ont à leur disposition une multitude de petits C.A.P. pouvant les accueillir en formation. Plus souvent lycéennes qu'apprenties, les filles sont aussi plus souvent confinées que les garçons dans des formations peu propices à la promotion professionnelle et sociale. Elles s'orientent ou sont orientées dans les grandes spécialités qui leur sont traditionnellement réservées comme celles de la coiffure et de la vente, et elles ont peu accès aux formations les plus récentes, même lorsque celles-ci n'exigent rien de spécifiquement physique que l'on pourrait associer à des dispositions plutôt masculines. Elles constituent le gros des populations dans les C.A.P. spécialisés dans l'accueil des jeunes en difficulté tels que ceux du nettoyage (Employé technique de collectivité, Maintenance et hygiène des locaux par exemple). Trois grandes spécialités préparées par apprentissage sont exclusivement féminines : Coiffure, Esthétique-cosmétique, Employé de pharmacie.

Bien que le nombre des C.A.P. scolaires soit moins élevé que celui des C.A.P. préparés par l'apprentissage, la diversité des premiers est plus grande puisque presque chaque spécialité dispensée en lycée professionnel se rapporte à un secteur d'activité particulier. Des C.A.P. de métier comme les C.A.P. de Coiffure ou d'Ébéniste côtoient des C.A.P. transversaux à plusieurs secteurs d'activité. Cette diversité semble tenir à la prégnance des logiques scolaires - comme la nécessité de scolariser les jeunes de l'enseignement adapté - réinterprétées au niveau local. C'est pourquoi le développement de certaines spécialités dans le système scolaire de formation est loin d'être systématiquement associé au développement des emplois (les professions de dessinateur, d'esthéticienne et de coiffeuse salariées n'ont cessé de réduire leurs effectifs entre 1990 et 1998, celles d'ouvriers qualifiés de type industriel stagnent ou régressent). De plus, si des possibilités d'emploi semblent ouvertes dans les professions de la sécurité, de la restauration collective, de la maintenance des bâtiments de collectivité ou du nettoyage industriel, qui voient se diffuser les sections de formation en lycée professionnel, rien n'assure que ces possibilités soient accessibles aux jeunes, puisque ces professions ont jusqu'à présent été caractérisées par la faible présence de la main d'ceuvre juvénile, et par la présence plus faible encore d'un personnel diplômé. 
Une étude sur les emplois de la sécurité (Pérez, 1998) et une autre sur ceux de la restauration collective (Mériot, 1997) soulignent ainsi le désavantage que constitue une trop grande jeunesse pour accéder à l'emploi.

Si le ministère de l'Éducation nationale s'est toujours interrogé sur le développement possible en lycée professionnel de diplômes créés en réponse à des demandes de certification de personnels non qualifiés ${ }^{9}$, la faible connaissance que l'on a des effets de ces expériences a ralenti leur multiplication. Formations aux contenus simples, mais qui accordent une place notable aux compétences relationnelles, ces C.A.P. sont réservés aux adultes en formation continue et aux jeunes en difficultés évincés des autres formations. Ils sont dirigés vers des emplois d'exécution longtemps nonqualifiés, qui sont devenus plus exigeants sous la pression des employeurs et des changements intervenus dans les organisations du travail des entreprises. Les compétences attendues des personnels dans ces professions sont désormais plus nombreuses. Comparés aux C.A.P. des métiers d'art, de l'alimentation, ou aux anciens C.A.P. industriels, ces diplômes apparaissent très différents : leurs contenus sont moins complexes et leurs savoirs moins importants. C'est au nom de ces particularités qu'ils ont été orientés vers l'accueil des publics en difficulté, mais rien ne nous permet aujourd'hui de mesurer l'efficacité d'une telle mesure. Bien que ces diplômes aient été institués à la demande de fédérations d'employeurs ou de grandes entreprises, le rôle du C.A.P. dans les emplois visés n'a pas une influence visible. Ces professions ont encore beaucoup recours à du personnel non-diplômé. Cependant, comme la formation continue occupe dans ces diplômes une place plus importante qu'ailleurs, on peut penser que la possession d'un diplôme devient peu à peu nécessaire pour occuper les emplois et que des chances de plus en plus nombreuses s'offriront aux futurs titulaires de ces C.A.P.

\section{Un diplôme difficile à obtenir}

Contrairement à ce que l'on entend dire parfois, le C.A.P. est loin d'être accordé à tous les candidats qui postulent. Dans de nombreux cas, il est même très difficile à obtenir. Les taux de réussite sont très variables entre les différentes spécialités et à l'intérieur d'un même secteur d'activité. Les C.A.P. dont les taux de réussite sont inférieurs à $70 \%$ sont les plus nombreux, ce qui contredit l'image de la faible sélectivité du C.A.P. Si l'entrée en formation se fait souvent en dehors de tout critère de sélection - ce qui n'est pas vrai pour tous les C.A.P. - l'accès au diplôme est, lui, beaucoup plus limité. Les C.A.P. qui affichent les meilleurs taux de réussite sont pour près d'un tiers d'entre eux « réservés " aux élèves de B.E.P. 18 C.A.P. sont présentés par une forte population d'élèves de B.E.P., présence qui contribue à cacher la faiblesse de la réussite des autres populations candidates.

Les apprentis semblent rencontrer plus de difficultés que les autres à obtenir le C.A.P., dans la mesure où ce sont surtout des C.A.P. préparés par la voie de l'apprentissage qui cumulent les taux les plus bas. Les C.A.P. où les adultes sont fortement représentés se démarquent également par leurs mauvais résultats. Cette réussite inégale des élèves, des apprentis et des adultes met en lumière l'avantage des populations lycéennes à l'égard d'un diplôme pourtant défini a priori par son profil peu scolaire.

La diversité d'origine des candidats à l'examen souligne la multitude des intérêts que suscite le C.A.P. Correspondant au premier niveau de la qualification professionnelle, le 
C.A.P. peut revêtir plusieurs sens, différents selon la population qui le convoite. Il peut être un simple diplôme de rattrapage pour les élèves formés au B.E.P., un diplôme indispensable et envié pour des salariés non qualifiés en voie de reconversion ou des demandeurs d'emploi, ou bien le diplôme minimum existant auquel peuvent prétendre les jeunes en difficultés scolaires.

Néanmoins, bien qu'elle soit à interpréter avec précaution, on peut voir sans risque d'erreur cette diversité comme le signe toujours plus affermi de la nécessité du diplôme. La multiplication des candidats issus de la formation continue pour l'accès à des professions traditionnellement ouvertes aux non-diplômés souligne à quel point la détention d'un diplôme est devenue une condition d'accès ou de maintien dans l'emploi, dans n'importe quelle profession que ce soit.

La liste suivante présente les 16 premiers C.A.P. en termes de populations candidates. Elle permet de mettre en oeuvre une comparaison entre les diplômes qui accueillent le plus grand nombre d'inscrits en formation et ceux dont les candidats sont les plus nombreux. On voit ainsi les différents usages qui reviennent à certains C.A.P., peu distribués dans le cadre de la formation initiale, mais largement répandus sur le marché du travail par l'intermédiaire des candidats libres et/ou des candidats adultes.

TABLEAU 2. Les seize plus gros C.A.P. par le nombre des candidats au diplôme

\begin{tabular}{|c|c|c|c|}
\hline & \multirow[t]{2}{*}{ CANDIDATS } & \multicolumn{2}{|c|}{ DONT, EN \% : } \\
\hline & & & FORMATION \\
\hline & & ISSUS & \\
\hline & & & CONTINUE \\
\hline & & DU B.E.P. & \\
\hline & & & ET CANDIDATS LIBRES \\
\hline 1 - Vente-relation clientèle & 31012 & $43 \%$ & \\
\hline 2 - Électrotechnique & 26570 & $84 \%$ & \\
\hline 3 - Petite enfance & 20263 & $42 \%$ & $46 \%$ \\
\hline $4-$ Cuisine & 17757 & $42 \%$ & \\
\hline 5 - Maintenance de véhicules particuliers & 14142 & $32 \%$ & $22 \%$ \\
\hline 6 - Coiffure & 12103 & $42 \%$ & \\
\hline 7 - Restaurant & 12047 & & \\
\hline 8 - Menuiserie-agencement & 9803 & $31 \%$ & \\
\hline 9-Pâtissier-glacier-chocolatier-confiseur & 7813 & $42 \%$ & \\
\hline 10 - Construction maçonnerie béton armé & 6168 & & \\
\hline
\end{tabular}




\begin{tabular}{|l|l|l|l|}
\hline $11-$ Carrosserie-réparation & 5699 & & \\
\hline $12-$ Boulanger & 5501 & $36 \%$ & \\
\hline $13-$ Finitions & 5210 & & \\
\hline $14-$ Installations sanitaires & 5206 & $17 \%$ & \\
\hline $15-$ Esthétique & 5197 & & $20 \%$ \\
\hline $16-$ Agent technique d'alimentation & 5089 & $76 \%$ & \\
\hline
\end{tabular}

TABLE 2. Sixteen larger C.A.P. according ta the number of candidates for the diploma

L'arrivée massive de candidats aux multiples origines à certains examens fait que des C.A.P. sans importance dans le système de formation occupent une place notable dans le système de certification. La position du C.A.P. Électrotechnique est ainsi dominante alors qu'il forme peu d'individus. Tous les C.A.P. auxquels se présentent d'importantes quantités d'élèves de B.E.P. se voient doter d'une influence supérieure à celle qui est la leur dans le système de formation. C'est vrai pour les C.A.P. du système scolaire comme pour les C.A.P. d'apprentis, puisque les jeunes formés en B.E.P. représentent une part importante de la population candidate dans de nombreux C.A.P.

41 Lorsqu'on regarde l'origine des candidats au C.A.P., on observe que ce diplôme reste marqué par sa population juvénile.

TABLEAU 3. Candidatures à l'examen du C.A.P. selon la filière d'origine (session 1996/97)

\begin{tabular}{|l|l|l|l|l|l|}
\hline ENSEMBLE DES SPÉCIALITÉS & SCOLAIRES & APPRENTIS & FORMATION & AUTRES & TOTAL \\
\hline$\%$ & 51 & 32 & 6,5 & 10,5 & 100 \\
\hline effectif & 141910 & 89218 & 17699 & 28472 & 277299 \\
\hline
\end{tabular}

Source : REFLET Céreq.

TABLE 3. Candidacies for the C.A.P. examination, according to scholastic origin (session 1996/97)

En réalité la part des scolaires est sur-représentée, près des deux tiers $(60 \%)$ des candidatures au C.A.P. sont le fait d'inscrits en cycle de B.E.P. pour lequel l'apprentissage reste encore une filière minoritaire.

TABLEAU 4. Candidatures à l'examen du C.A.P. selon le cycle de formation (session 1996/97)

\begin{tabular}{|l|l|l|l|l|l|l|l|}
\hline ENSEMBLE & UNITÉS & C.A.P. & C.A.P. & C.A.P. & C.A.P. & & \\
\hline & & & & &. & .B E.P. & TOTAL \\
\hline DES SPÉCIALITÉS & CAPITAL. & 1 AN & 2 ANS & 3 ANS & NON PRÉCISE & & \\
\hline
\end{tabular}




\begin{tabular}{|l|l|l|l|l|l|l|l|}
\hline$\%$ & 3 & 1 & 13 & 5 & 18 & 60 & 100 \\
\hline effectif & 4895 & I 043 & 22446 & 9113 & 32602 & 106378 & 176477 \\
\hline
\end{tabular}

Source : REFLET Céreq.

TABLE 4. Candidacies for the C.A.P. examination according to the levet of formation (session 1996/97)

\section{Le C.A.P. dans l'ensemble des emplois occupés ${ }^{10}$}

À une date donnée le stock d'une catégorie de diplômés dans la population active est le résultat de la confrontation au cours du temps des politiques successives de création / rénovation des diplômes et de l'usage que les acteurs (élèves et employeurs) font de ces diplômes. Les changements intervenus dans la structure des diplômés de l'enseignement professionnel au niveau $\mathrm{V}$ entre 1990 et 1998 montrent que les évolutions peuvent s'avérer sensibles sur courte période,

TABLEAU 5. Diplômés de l'enseignement technique et professionnel

\begin{tabular}{|l|l|l|l|l|l|l|l|l|}
\hline & & 1990 & & 1992 & & 1995 & & 1998 \\
\hline Certif. éduc. profession. & & 0,50 & 0,90 & 0,80 & 0,76 \\
\hline C.A.P. ou B.E.P. par appr. & 11,60 & 22,50 & 23,30 & 24,00 \\
\hline C.A.P. hors apprentissage & 44,20 & 31,80 & & 29,20 & 27,46 \\
\hline B.E.P. hors apprent. & 16,00 & & 16,30 & & 18,60 & 18,79 \\
\hline Autres dip, niv, V & & 6,80 & & 6,70 & & 6,30 & 5,96 \\
\hline Bt, Btn & 12,20 & & 13,80 & & 13,30 & 12,98 \\
\hline Bac pro & 11,10 & & 2,30 & & 3,20 & 4,25 \\
\hline Autre dip, niv, IV & 400135 & 12 & 404938 & 130 & 136411 & 816203 \\
\hline Brevet d'ens. agri., com., hôt. & 2,80 & & 2,50 & & 2,40 & 2,25 \\
\hline Total \% \\
Effectifs
\end{tabular}

Source : Enquête annuelle sur l'Emploi, I.N.S.E.E.

TABLE 5. Graduates of vocational education and training

En 1990, pour près de la moitié ( 45 \%) de la population active ayant déclaré posséder un diplôme de l'enseignement technique et professionnel, le C.A.P. préparé hors apprentissage (donc essentiellement par la voie scolaire, avant l'entrée dans la vie active) était le diplôme le plus élevé possédé. Huit ans plus tard, les effets cumulés des 
mesures d'orientation en $5 \mathrm{e}$ et de la loi de 1987 sur l'apprentissage modifient substantiellement la structure du stock de diplômés de niveau V. Le C.A.P. hors apprentissage ne concerne plus que $27 \%$ de la population. L'évolution est à l'avantage du B.E.P. qui « profite » à la fois d'un développement en milieu scolaire (+ 4 points) et de l'ouverture de la filière apprentissage.

Dans l'ensemble des titulaires, au sein de la population active, d'un C.A.P. obtenu hors apprentissage, on perçoit les effets des réformes de la scolarité au niveau du collège et du processus d'orientation en classe de cinquième. La part des titulaires d'un C.A.P. en trois ans après la cinquième ne cesse de baisser sur la période. Cette évolution tient d'une part à l'entrée en activité de jeunes dotés de plus en plus souvent d'un C.A.P. post troisième, titulaires du B.E.P.o (ou B.C.), et d'autre part à la sortie d'activité des générations ayant obtenu le diplôme à une époque où le modèle était celui du C.A.P. traditionnel en trois ans.

L'accès à un autre diplôme de l'enseignement général pour des titulaires d'un C.A.P. hors apprentissage reste minoritaire, autour de $3 \%$.

Compte tenu du mode de tri contraint par l'enquête Emploi qui ne permet pas d'isoler l'ensemble des C.A.P., les cas de double certification C.A.P. et B.E.P. ne sont pas pris en compte. Il s'agit ici en effet d'individus n'ayant que

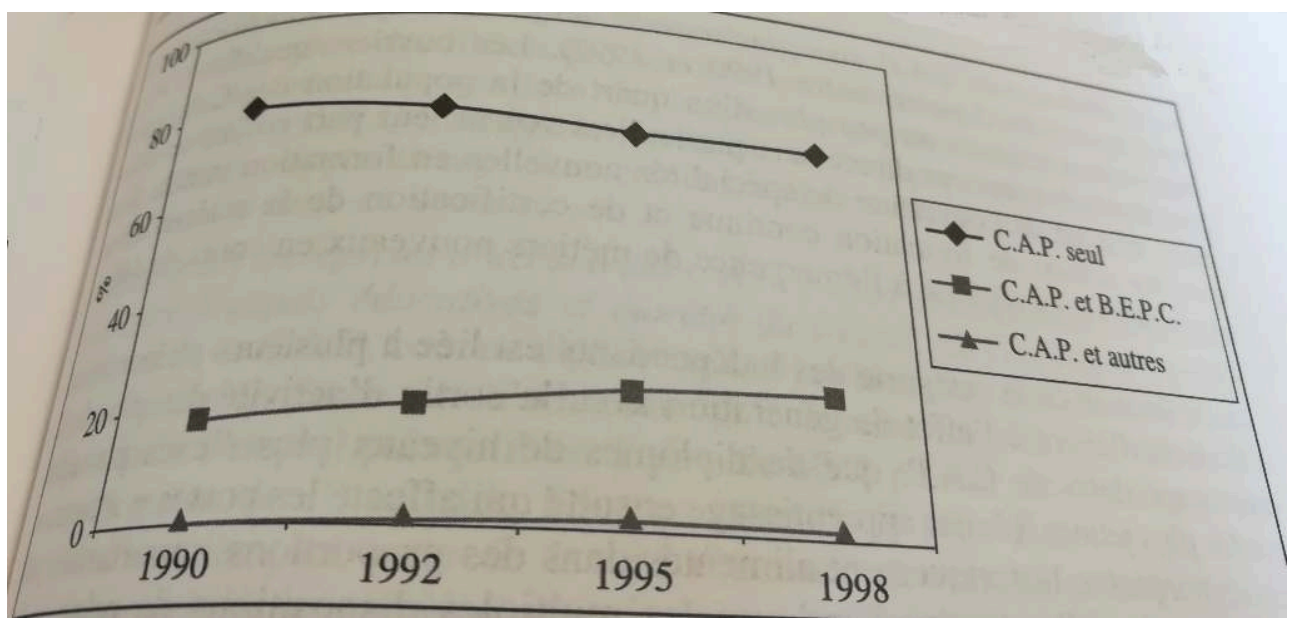

leSource : Enquête annuelle sur l'Emploi, I.N.S.E.E.

C.A.P. hors apprentissage, comme diplôme technique ou professionnel le plus élevé possédé.

Dans une perspective de " concurrence " entre diplômes, de même niveau ou de niveaux différents, nous avons tenté d'identifier des liaisons entre les parts respectives des C.A.P. étudiés et celles d'autres diplômes ou niveaux de formation (dans le cas de concurrence potentielle de non diplômés), au sein des secteurs. Ceci sans préjuger du sens des liaisons éventuelles, la concurrence pouvant générer des phénomènes de substitution ou de complémentarité. Une forte part de niveaux IV dans un secteur peut en effet renforcer la place faite au C.A.P. réservé dans ce cas à des catégories d'emplois particulières, alors qu'une prédominance du niveau $\mathrm{V}$ peut être le résultat d'une substitution quasi systématique du C.A.P. par le B.E.P. Pour 1990, il n'apparait pas de phénomène marquant, si ce n'est une corrélation nette entre la part des C.A.P. hors apprentissage et la part de l'ensemble niveau $\mathrm{V}$ dans les secteurs. Dans le Bâtiment où 
un quart de la population occupée en 1990 possédait un C.A.P. hors apprentissage, la moitié de la main-d'oeuvre ne possédait aucun diplôme professionnel.

En 1997 malgré des proportions de C.A.P. hors apprentissage et de l'ensemble niveau V dans l'emploi en baisse par rapport à 1990 (moins $9 \%$ et moins $3 \%$ respectivement), la liaison existe toujours entre les deux indicateurs mais avec une moindre évidence. Les C.A.P. étudiés sont toujours d'autant plus présents dans les secteurs où le niveau $\mathrm{V}$ est davantage fréquent, mais avec la recomposition de ce dernier ils perdent de l'importance au profit des deux autres diplômes/ filières de formation de ce même niveau (B.E.P. hors apprentissage, C.A.P. ou B.E.P. obtenus par apprentissage). La part du niveau IV (Btn, bacpro, bt) ne progresse pas. Il n'apparaît pas de lien entre ce sousensemble et les C.A.P. étudiés, lien qui pourrait par exemple révéler des politiques sectorielles mises en oeuvre après 10 ans de vie du bac pro.

La caractérisation de la population de référence au regard des catégories socioprofessionnelles (C.S.P.) et des secteurs et entreprises d'emploi identifie plusieurs phénomènes entre 1990 et 1997.

55 Les titulaires du C.A.P. sont plus souvent, au fil du temps, dans la catégorie « employés » (+ 5 points entre 1990 et 1997). Les ouvriers qualifiés (0.Q.) représentent toujours un peu plus d'un quart de la population des C.A.P. Les personnels des services directs aux particuliers voient leur part croître sensiblement, résultat de l'ouverture de spécialités nouvelles en formation initiale ainsi que des actions de formation continue et de certification de la main-d'œuvre expérimentée en réponse à l'émergence de métiers nouveaux en cours de définition.

56 La diminution de la catégorie des indépendants est liée à plusieurs phénomènes. Elle tient d'abord à l'effet de génération avec la sortie d'activité des plus âgés davantage dotés de C.A.P. que de diplômes de niveaux plus élevés possédés par les plus jeunes. L'effet apprentissage ensuite qui affecte les postes " artisan ", " commerçant ", historiquement alimentés dans des proportions importantes par l'apprentissage. Il est normal qu'avec les multiples dispositions de relance de l'apprentissage, y compris l'ouverture au B.E.P., le C.A.P. voie scolaire y soit moins représenté.

Les mêmes phénomènes pourraient expliquer la moindre représentation des O.Q. type artisanal. Il n'y a pas d'exception à la diminution de la part des diplômés du C.A.P. entre 1990 et 1997. Le phénomène vaut pour chacune des C.S.P. La diminution est d'autant plus forte que le C.A.P. était bien représenté dans les catégories.

Les C.A.P. sont concentrés dans quatre secteurs : Services marchands, Services non marchands, Commerce, Bâtiment et génie civil. Avec quelques modifications de rang ces quatre secteurs pèsent à peu près le même poids entre les deux dates $(58 \%$ de la population active occupée dotée d'un C.A.P. en 1990, 56 \% en 1997). L'évolution sectorielle la plus significative est celle des industries agro-alimentaires (I.A.A.) qui occupent $11 \%$ de la population étudiée, contre $3 \%$ seulement en 1990. Un processus de qualification de la main-d'œuvre (longtemps sans aucune formation professionnelle) a été mis en œuvre dans ce secteur, tant au niveau du recrutement (avec l'embauche de titulaires de C.A.P. délivrés par l'enseignement agricole) qu'au niveau de la certification de la main-d'oeuvre occupée (contrats de qualification pour les jeunes, C.Q.P. pour les moins jeunes) ${ }^{11}$.

59 Les ouvriers (qual(és et non qualifiés) sont de plus en plus souvent en activité dans des entreprises du secteur tertiaire (respectivement $39 \%$ en 1990 et $43 \%$ en 1997, $37 \%$ en 
1990 et $45 \%$ en 1997). Dans le secteur industriel les I.A.A. mobilisent en $199716 \%$ des O.Q. titulaires de C.A.P. (4 \% seulement en 1990). On observe une évolution inverse (16 $\%$ en 1990 et $4 \%$ en 1997) dans les industries de biens d'équipement, secteur en crise depuis plusieurs années et qui procède à des réductions drastiques d'effectifs pour certaines catégories de main-d'oeuvre. Celle des techniciens (31\% en 1990 contre $6 \%$ seulement en 1997) en serait une conséquence manifeste. Elle correspond à la sortie d'activité des plus âgés promus dans le cadre des marchés internes à partir de la possession d'un C.A.P. à une époque où ce diplôme constituait un point d'ancrage dans des filières promotionnelles. Le bâtiment mobilise toujours à peu près les mêmes proportions de chacune des grandes C.S.P. (techniciens/ maîtrise, O.Q. et 0.N.Q.), malgré les fortes baisses de la population des diplômés de C.A.P. hors apprentissage. Les artisans titulaires de ce diplôme sont pour moitié environ dans ce secteur.

La taille des entreprises d'accueil des C.A.P. : reflet de confrontations successives entre logiques éducatives et marché du travail. Si l'on retient l'image traditionnelle du C.A.P. comme diplôme centré sur le métier, dans des entreprises de type artisanal, force est d'admettre qu'il assume toujours sa fonction de préparation à l'emploi puisqu'il est à plus de $40 \%$ présent dans les entreprises occupant moins de 10 salariés. Pour un quart de la population il s'agit d'une activité d'auto emploi, exercée à titre d'artisan, sans aucun salarié. Mais en même temps un tiers environ des titulaires du C.A.P. hors apprentissage se retrouvent dans les entreprises de 100 salariés ou plus ( $18 \%$ dans des entreprises de plus de 1000 salariés). Le phénomène est explicable pour partie par le fait que les générations entrées plus récemment sur le marché du travail avec un niveau de formation (et de diplôme) plus élevé que leurs aînés sont embauchées surtout par de petites et moyennes entreprises.

61 L'analyse ci-dessus s'appuie sur des données de stocks qui sont la résultante d'une évolution de longue période des rapports entre appareils éducatif et productif. Les interprétations avancées font référence à plusieurs reprises aux flux qui les ont générées, en particulier aux flux d'entrée en activité de jeunes diplômés qui font l'objet de l'analyse qui suit.

\section{Les modalités d'accès des titulaires du C.A.P. au marché du travail}

62 Basée sur des exploitations de l'enquête «jeunes et carrières » de mars $1997{ }^{12}$, cette analyse est conduite selon une perspective inter-générationnelle, avec en arrière-plan l'hypothèse d'une dégradation progressive des conditions d'accès aux emplois " cibles » et de maintien sur le marché du travail, tout en intégrant des éléments d'appréciation liés à la plurifonctionnalité actuelle du C.A.P.

\section{Caractérisation des situations au premier emploi de plus de 6 mois}

Comme le proposait l'Insee lors d'une exploitation de l'enquête portant sur l'ensemble des diplômes (cf. Insee première $\mathrm{n}^{\circ} 598$ - juillet 1998), nous avons construit trois " générations » « $78 »$, «88 », «93» composées des individus ayant terminé leurs études respectivement en 77-78-79, 87-88-89, et 92-93-94. 

serait à une moindre accessibilité à un premier emploi qualifié, au moins pour les ouvriers. L'interprétation des résultats pour les autres C.S.P. reste délicate, la nomenclature utilisée ne distingue pas les emplois qualifiés des emplois non qualifiés pour la catégorie "employés ». Si les entrants en activité à la fin des années 80 s'insèrent davantage que leurs prédécesseurs en situation " d'employés ", cela ne dit rien sur le degré de qualification des emplois correspondants. Une analyse plus fine, au moyen d'une variable construite manuellement par les gestionnaires de l'enquête ${ }^{13}$, montre que la croissance de la part de la catégorie «employés » pour la génération 88 repose en partie sur un accès plus fréquent que dans le cas de la génération précédente à des emplois non qualifiés des services (agent de surveillance, aide à domicile, agent d'entretien, employé de maison, etc.). Le détail des professions exercées au sein des grandes C.S.P. indique qu'à l'issue d'un C.A.P. l'insertion (au sens de l'obtention d'un premier emploi de plus de 6 mois) sur le marché du travail a lieu majoritairement dans des emplois ouvriers, qualifiés ou non qualifiés. Cette prépondérance de l'emploi ouvrier traverse cependant des évolutions fortes de la structure de cette catégorie. Les emplois ouvriers de type industriel qui accueillaient un tiers de la génération 78 accueillent un quart seulement des deux autres générations. La part des ouvriers non qualifiés reste stable au cours du temps ( $18 \%$ environ des premiers emplois) mais celle des emplois qualifiés n'est plus que de $7 \%$ au début de la décennie 90 , contre $15 \%$ pour les deux précédentes. résulte à la fois d'une hausse de l'emploi qualifié (de 20 à $31 \%$ ) et de l'emploi non qualifié (de 9 à $15 \%$ ). Une enquête réalisée par le Céreq en 1993 révélait que les emplois occupés par les débutants sont en lien étroit avec leur formation initiale lorsque celleci relève du secteur des métiers où le C.A.P. domine largement voire même est le seul diplôme de niveau V (Bref $\mathrm{n}^{\circ} 144$ - juillet 1998). L'analyse confirme également la difficulté, croissante d'une génération à l'autre, d'accéder, avec un simple C.A.P., à des emplois qualifiés administratifs ou commerciaux d'entreprises, d'où le repli vers des emplois relevant $\mathrm{du}$ domaine des services aux particuliers, emplois souvent non qualifiés, aux contours non stabilisés et/ou en cours de professionnalisation.

Du seul point de vue statistique, la dégradation, avec le temps, des conditions d'accès au marché du travail et de stabilisation dans l'emploi est flagrante. À la fin des années 70 les $2 / 3$ des entrants sur le marché du travail avec un C.A.P. obtenaient leur premier emploi de plus de 6 mois au moyen d'un contrat à durée déterminée (C.D.I.). Un peu plus d'un tiers (36\%) sont dans cette situation au début de la décennie 90 . L'ensemble C.D.I.C.D.D. (contrat à durée déterminée) ne représente plus que $60 \%$ des modalités d'emploi, contre 79 \% 15 ans auparavant. Les débutants s'insèrent de plus en plus souvent sur le marché du travail avec des statuts précaires, en situations de stagiaires, au moyen de mesures d'aide.

On peut avoir une autre lecture des résultats observés si l'on tient compte du phénomène d'hétérogénéisation de la population des titulaires d'un C.A.P., diplôme de plus en plus considéré comme un objectif de certification minimale pour des jeunes en difficultés dans l'appareil scolaire ${ }^{14}$

\begin{tabular}{|l|l|l|l|}
\hline ORIGINE SCOLAIRE DES ÉLÈVES EN ... & C.A.P. 2 ANS & C.A.P. 3 ANS & ENSEMBLE C.A.P. \\
\hline
\end{tabular}




\begin{tabular}{|l|l|l|l|l|}
\hline $3^{*}$ générale ou techno. & 12915 & 753 & 13668 & $42 \%$ \\
\hline r, 1', Terminale gén. ou techn. & 3346 & 71 & 3417 & $10 \%$ \\
\hline B.E.P. & 1031 & 44 & 1075 & $3 \%$ \\
\hline Enseignement adapté & 9400 & 1971 & 11371 & $35 \%$ \\
\hline e, 5', 4' & 239 & 2385 & 2624 & $8 \%$ \\
\hline Divers & 167 & 342 & 509 & $2 \%$ \\
\hline
\end{tabular}

(Source : D.P.D., élèves en première année de C.A.P., rentrée 98)

À dix ans d'intervalle la dégradation des conditions de la transition « formation-marché du travail " pour les titulaires de C.A.P. est perceptible. La part des actifs occupés diminue de 4 points, le taux de chômage passe de 6,5 à 8,5\%. Compte tenu des observations disponibles dans l'enquête une analyse au niveau des spécialités de formation d'origine des titulaires de C.A.P. ${ }^{16}$ n'est possible que pour deux groupes seulement : " agro-alimentaire, alimentation, cuisine » et «mécanique générale et de précision, usinage ».

74 spécialités entre les deux générations retenues est à peine supérieure à celle de la moyenne des C.A.P., toutes spécialités confondues, même si le niveau de l'emploi était 
et demeure plus élevé qu'en moyenne. Par contre, dans chacun des deux groupes, la dégradation s'accompagne d'un retrait du marché puisque l'inactivité croît, contrairement à ce que l'on observe pour l'ensemble des spécialités.

Cinq domaines de spécialités sont également suffisamment représentés pour faire l'objet d'un traitement particulier : transformation, génie civil-construction et bois), mécanique-électricité-électronique, échange et gestion, services aux personnes.

Le contraste est flagrant entre les domaines de spécialités secondaires et tertiaires. La part des individus en emploi 7 ans après la fin des études diminue d'une génération à l'autre dans les premiers, elle croît (même si la croissance reste faible dans le domaine des services aux personnes) dans les seconds où elle était la plus faible en 1978.

La part des chômeurs crô̂t fortement dans les spécialités du domaine de la " transformation ", et du " génie civil, construction et bois ». Dans les deux domaines tertiaires où il était minoré par une forte inactivité féminine dans la génération 78 le chômage croît en 1988 malgré...ou à du fait de ...l'existence de meilleures opportunités d'emploi. La situation reste stable pour les sortants des spécialités relevant de la mécanique et de l'électricité.

Globalement la situation à D+7 s'est améliorée par rapport aux premiers emplois de plus de 6 mois. Les actifs occupés sont davantage en position d'ouvriers qualifiés ou de maitrise qu'en début d'activité. Le constat vaut pour les deux générations d'entrants sur le marché avec un C.A.P., même si l'ascension vers des catégories intermédiaires (entre exécution et encadrement) est plus limitée dans la décennie 90 que dans la décennie précédente. Ce qui pourrait justifier un repli vers des situations professionnelles « autres ».... que le salariat (indépendants notamment). En premier emploi la croissance de la part de la catégorie " employés " pour la génération 88 reposait en partie sur un accès plus fréquent que pour la génération 78 à des emplois non qualifiés des services (agent de surveillance, aide à domicile, agent d'entretien, employé de maison, etc.). La tendance se confirme pour les situations respectives à D+7.

79 Si les " employés " représentent toujours un tiers environ des emplois pour les deux générations, la structure interne des emplois de la catégorie évolue plutôt défavorablement pour les cohortes entrées plus récemment sur le marché. Le maintien dans l'emploi se fait de plus en plus sur des emplois non qualifiés qui sont presque aussi fréquent que les emplois qualifiés ( $46 \%$ et $54 \%$ respectivement) ${ }^{17}$.

80 Au niveau des spécialités seules les données relatives au groupe 221 (" agroalimentaire, alimentation, cuisine ») présentent quelques garanties de représentativité. 7 ans après la fin des études sanctionnées par un C.A.P. dans ce groupe de spécialités les individus entrés sur le marché à la fin des années 80 sont davantage ouvriers qualifiés que leurs prédécesseurs 10 ans auparavant dont une plus grande part restait classés O.N.Q. Ce qui les différencie c'est aussi une plus grande difficulté pour les plus jeunes à progresser (à partir d'un C.A.P.) dans une carrière débouchant sur des emplois de maîtrise ou de technicien, du fait probablement d'une concurrence accrue avec des entrants de niveau plus élevé, de plus en plus nombreux.

81 Pour les domaines de spécialités suffisamment représentés dans l'échantillon, les positions professionnelles des actifs occupés à l'issue de 7 années de présence sur le marché du travail sont contrastées et parfois très éloignées des valeurs moyennes pour l'ensemble des populations dans chacune des générations. Des « effets spécialités » sont identifiables. 7 ans après la fin des études initiales, on occupe plus fréquemment des 
emplois d'O.N.Q. si l'on appartient à la génération 88 (par comparaison avec les aînés de 78), tant en " génie civil, construction et bois " ou en " mécanique-électricitéélectronique ", qu' à partir d'une formation du domaine tertiaire " échange et gestion " où il devient difficile avec le temps de conserver une position d'employé.

Les situations respectives sont inversées pour la " transformation " où l'on est davantage qualifié dans les emplois d'exécution mais où la progression vers des postes de maîtrise est moindre pour la génération plus jeune.

L'évolution plus favorable dans le domaine des « services aux personnes » serait liée au mouvement de professionnalisation et de qualification des emplois (moins d'ouvriers, non qualifiés notamment, et davantage d'employés) ainsi qu'à l'émergence de postes d'encadrement accessibles à une population ayant acquis un minimum d'expérience professionnelle à partir de la détention d'un C.A.P. obtenu en formation initiale.

\section{Conclusion}

L'observation des événements qui ont émaillé l'histoire du C.A.P. confirme que le devenir d'un diplôme ne dépend pas exclusivement des décisions prises par la " machinerie sociale " qui, au nom du paritarisme, édicte ce qui à un moment donné constitue la norme en matière de relation entre formation et emploi. Au-delà des décrets, la vie du diplôme est aussi ce qu'en font effectivement les agents : responsables d'établissements, candidats à la formation et à la certification, employeurs. Malgré plusieurs disparitions programmées, ce diplôme a résisté, grâce notamment au " poids sur le marché de l'emploi des petites entreprises, en particulier dans des secteurs à dominante artisanale comme le bâtiment, pour qui ce type de qualification centrée sur l'accumulation de savoir-faire demeurait une nécessité vitale... le C.A.P. a été officiellement rétabli en 1971 » (Pelpel \& Troger, 1993, p. 132). Cette caractérisation de l'usage qui est fait du C.A.P. par l'appareil productif est confirmée pour les années récentes.

85 L'analyse de l'insertion des titulaires de C.A.P. a montré que l'accès aux emplois ouvriers qualifiés de type industriel est de plus en plus difficile pour les générations successives. Si l'insertion sur des emplois ouvriers demeure néanmoins majoritaire c'est de plus en plus souvent dans des structures productives de type artisanal. La situation 7 ans après la fin des études initiales témoigne de la dégradation, d'une génération à l'autre, des conditions de stabilisation sur le marché du travail. Le maintien dans des emplois de la catégorie "employés ", qui accueille plus souvent des entrants, se fait au prix d'une déqualification croissante. Ces observations sont à nuancer seulement pour quelques domaines où des mouvements de professionnalisation en cours offrent des opportunités de maintien dans (ou de progression vers...) des postes qualifiés.

La situation actuelle du C.A.P. est autant le fait du système éducatif que celui du marché du travail qui aurait plutôt, au moins pendant un temps et sur des segments bien particuliers, retardé sa désaffection. L'officialisation d'une fonction de remédiation pour des jeunes en difficultés dans le système scolaire reste malgré tout pour le système éducatif une décision délicate à adopter. D'un point de vue opérationnel, l'identification de C.A.P. plus propices que d'autres à l'accueil en formation de jeunes en difficultés pose problème. L'obtention du diplôme demeure relativement difficile. En outre elle ne garantit pas une insertion professionnelle favorable. Les jeunes les plus en 
difficultés sont orientés traditionnellement vers les C.A.P. les moins attractifs (bâtiment gros œuvre, serrurerie-métallerie, nettoyage industriel, vente), réputés pour être faciles, mais présentant des taux de réussite inférieurs à $70 \%$. Restent, pour l'industrie, les C.A.P. construits pour la certification des ouvriers spécialisés, dont l'emploi subsisterait sous des formes nouvelles. Mais le faible attrait des métiers industriels auprès des jeunes ne permet pas d'y voir un créneau très porteur. Ce sont surtout des C.A.P. non marqués par l'histoire, non reliés à un corps particulier de professionnels (comme l'étaient les C.A.P. d'ajusteur ou de tourneur, par exemple), associés à des professions émergentes ou en quête d'une meilleure image et d'une meilleure légitimité sociale (comme celles de la vente et de la distribution), qui peuvent répondre aux attentes de formation et d'emploi des jeunes caractérisés par leurs difficultés. De tout petits C.A.P., gérés localement au moyen de partenariats écoles/ entreprises, revêtent sans doute plus d'intérêt que les C.A.P. à gros flux dans lesquels on oriente habituellement les jeunes en difficultés. Mais alors, dans cette perspective, quel chemin parcouru depuis les origines du diplôme géré initialement au plan local pour une certification des apprentis !

\section{BIBLIOGRAPHIE}

Bouyx, B. (1997). L'enseignement technique et professionnel. Paris : la Documentation Française.

Brucy, G. (1989). C.A.P. et certificats de spécialité : les enjeux de la formation au lendemain de la deuxième guerre mondiale. Formation-Emploi, 27/28, 131-146.

Brucy, G. (1998). Histoire des diplômes de l'enseignement technique et professionnel (1880-1965). L'État, l'École, les Entreprises et la certification des compétences. Paris : Belin.

Charlot, B., \& Figeat, M. (1985). Histoire de la formation des ouvriers : 1789-1984. Paris : Éditions Minerve.

Fourcade, B., \& Ourtau, M. (1999). Le B.E.P. : un diplôme, deux finalités ? Formation- Emploi, 66, 39-55.

Maillard, F. (1992). Les emplois de la vente : quelle place pour le C.A.P. et le B.E.P. ? C.P.C./ Document, 92/6, 1-100.

Maillard, F. (1994). C.A.P.-B.E.P. : association ou concurrence ? Le cas des formations de la vente. Formation-Emploi, 47, 59-72.

Mériot, S. (1997). Restauration collective : analyse des besoins de formation pour une rénovation des diplômes. C.P.C./Document, 97/6, 1-116.

Pelpel, P., \& Troger, V. (1993). Histoire de l'enseignement technique. Paris : Hachette Éducation.

Pérez, D. (1998). Les métiers et les formations de la sécurité. C.P.C./Document, 98/7, 1-114.

Tanguy, L. (1991). Quelle formation pour les ouvriers et les employés en France? Paris : La

Documentation Française. 


\section{NOTES}

1. Les rappels historiques s'inspirent très largement des analyses proposées par B. Bouyx (1997),

G. Brucy $(1989,1998)$, P. Pelpel \& V. Troger (1993).

2. Malgré la réforme Haby de 1977 et le passage à une logique de recrutement vers l'enseignement professionnel à partir de la troisième (le B.E.P., Brevet d'Études Professionnelles, a été créé entre temps, en 1966) dans des lycées d'enseignement professionnels (L.E.P.) remplaçant les CET., la première orientation vers le C.A.P. en trois ans à l'issue de la classe de $5^{*}$ reste la norme jusqu'en 1980.

3. Cf. Un lycée pour le XXI` siècle : l'enseignement professionnel intégré, conférence de presse du 24 juin 1999. M.E.N.R.T.

4. À partir du fichier REFLET du C.E.R.E.Q.

5. En 1966 ; l'insuffisance du CAR était déjà dénoncée à ce moment, au nom des évolutions technologiques et de l'organisation du travail dans les entreprises. Le C.A.P. devait en conséquence être réservé aux apprentis, censés se préparer à l'exercice de métiers peu soumis au changement. A contrario, le B.E.P. proposait une formation plus polyvalente, construite sur une plus grande part d'enseignement général et sur un meilleur niveau scolaire d'origine.

6. Opus cité.

7. Cependant, le nombre de C.A.P. peut varier de manière considérable selon que l'on prend ou non en compte les options des diplômes. Dans la mesure où, dans la période de référence, presque chaque C.A.P. compte de 3 à 5 options, le total des C.A.P. peut plus que doubler. Ce comptage est d'autant plus difficile à faire que certaines options peuvent n'être que des formes légères de spécialisation, alors que d'autres distinguent des métiers, des produits et des secteurs d'activité.

8. «Repères et Références statistiques ", édition 1998. Ministère de l'Éducation nationale, de la Recherche et de la Technologie.

9. Une étude du C.E.R.A.F. a été consacrée à ce sujet en 1995, pour ce qui concerne les C.A.P. industriels : « Les C.A.P. industriels : de la formation continue vers la formation initiale », C.P.C./ Document $n^{\circ} 95 / 7$. Les conclusions de ce travail incitent à la prudence.

10. Les analyses proposées ici ont été réalisées à l'occasion d'une convention d'étude passée entre la D.E.S.C.O. et le Lirhe sur la réalité du C.A.P. aujourd'hui. Les données détaillées figurent dans le rapport d'étude.

11. Cf. les travaux du Céreq sur les C.Q.P., documents synthèse $n^{\circ} 132$, mars 1998.

12. Le tri de la population de référence (les titulaires d'un C.A.P. comme diplôme le plus élevé obtenu) a été opéré à partir des libellés bruts déclarés dans l'enquête, dans la base de donnée E.T.D.I.P. Tous les C.A.P. sont donc retenus, sans tenir compte de la filière suivie pour l'accès au diplôme. Dans ce cas les apprentis font partie de la population étudiée.

13. Construction basée sur les définitions utilisées par Bisault, Destival et Goux (« emploi et chômage des non qualifiés en France ». Économie et statistique, n²73, 1994-3).

14. D'un point de vue méthodologique plus général ceci soulève le problème de la signification des comparaisons inter-générationnelles en référence aux diplômes. Audelà des effets conjoncturels (...de moment ?)

15. Nous ne disposons pas d'un recul suffisant pour étudier la situation de la génération « 93 » à D+7 (où D est la date de fin d'études).

16. Nous avons construit, à partir des libellés bruts des C.A.P. déclarés dans l'enquête, une variable «diplo » dont le codage se réfère à celui qui a été retenu dans la nomenclature des spécialités de formation du C.N.I.S. de 1993. 
17. Ce résultat doit être interprété avec précaution du fait qu'il repose largement sur une partition très empirique de l'ensemble de la catégorie « employés »•

\section{RÉSUMÉS}

En s'appuyant sur le cas particulier du C.A.P. cet article est une réflexion plus générale sur la nature des diplômes professionnels. L'approche historique du processus de création, rénovation et suppression des C.A.P. témoigne du caractère évolutif d'un diplôme professionnel dans sa fonction première de préparation à l'accès à l'emploi. Elle met l'accent sur l'interdépendance des diplômes et sur les risques d'affaiblissement, le cas échéant, du rôle joué par chacun d'eux. Elle analyse l'offre actuelle de formation et de certification en C.A.P., ainsi que les ajustements opérés sur le marché du travail. Les auteurs concluent à une plus ou moins grande capacité du diplôme, selon les périodes, à concilier les logiques différentes qui président à la fois à sa conception et aux usages qui en sont faits par les systèmes éducatif et productif.

Based on the particular case of the C.A.P. this article provides a more general reflection on the nature of professional diplomas. An historical approach to the process of creation, restoration and removal of the C.A.P. shows the evolutionary character of a professional diplorna in its primary function as a means of preparation to employnent access. It stresses the interdependence of diplomas and, in some cases, the risks of diminution of the rote played by each one of them. It studies the current offer in C.A.P. formation and certification, as well as the adjustments related to the labour market. The authors conclude that, depending on the time period, die diploma may be more or less successful in reconciling Me different objectives which govern its conception, and Us use by educational and production systems.

INDEX

Keywords : Training, diploma, certification, transition

Mots-clés : Formation professionnelle, diplôme, certification, insertion

\section{AUTEURS}

\section{ÉRIC CAHUZAC}

est Ingénieur I.N.R.A., à l'Unité d'Économie et Sociologie Rurale, à Toulouse.

\section{FABIENNE MAILLARD}

est Chargée d'études à la Direction des Enseignements Scolaires (D.E.S.C.O.) du M.E.N.R.T., à Paris.

\section{MAURICE OURTAU}

est Ingénieur C.N.R.S. au Laboratoire Interdisciplinaire de Recherche sur les Ressources Humaines et l'Emploi (L.I.R.H.E.), à l'Université des Sciences Sociales de Toulouse. Laboratoire Interdisciplinaire de Recherche sur les Ressources Humaines et l'Emploi. Université des Sciences 
sociales, place Anatole-France, 31042 Toulouse cedex. Tél. : 05-61-63-38-70. Fax : 05- 61-63-38-60.

E-mail : ourtau@univ-tiselfr 